\title{
Spinor Two-Point Functions in Maximally Symmetric Spaces
}

\author{
B. Allen ${ }^{1}$ and C. A. Lütken ${ }^{2}$ \\ 1 Department of Physics, Tufts University, Medford, MA 02155, USA \\ 2 Center for Theoretical Physics, University of Texas, Austin, TX 78712, USA
}

\begin{abstract}
The two-point function for spinors on maximally symmetric fourdimensional spaces is obtained in terms of intrinsic geometric objects. In the massless case, Weyl spinors in anti de Sitter space can not satisfy boundary conditions appropriate to the supersymmetric models. This is because these boundary conditions break chiral symmetry, which is proven by showing that the "order parameter" $\langle\overline{\psi \psi}\rangle$ for a massless Dirac spinor is nonzero. We also give a coordinate-independent formula for the bispinor $S(x) \bar{S}\left(x^{\prime}\right)$ introduced by Breitenlohner and Freedman [1], and establish the precise connection between our results and those of Burges, Davis, Freedman and Gibbons [2].
\end{abstract}

\section{Introduction}

Maximally symmetric spacetimes provide an interesting background for studying quantum field theory in curved space. They also have nice applications: de Sitter space (DS) appears in "inflationary" models of the early universe [3], and anti de Sitter space (ADS) as the classical ground state of gauged supergravity models [1].

In this paper we extend the coordinate independent construction of two-point functions for bosons [4] to the fermionic case. The method employs only geometric quantities intrinsic to the manifold, such as the propagator of parallel transport. By exploiting the maximal symmetry of the spacetime we therefore obtain very simple expressions.

We use notation in which spinors $\xi^{A}$ (conjugate spinors $\bar{\xi}^{A}$ ) have undotted (dotted) capital latin indices. This notation is explained in [5], and restricts the applicability of this work to four dimensions.

In Sect. two we introduce the parallel propagator for spinors and calculate its covariant derivative. Section three uses this result to find the massive two-point functions for DS and ADS. Section four treats the massless limits, and shows why chiral symmetry must be broken in ADS. In Sect. five we obtain a simple formula for the Killing bispinor $S(x) \bar{S}\left(x^{\prime}\right)$ introduced in $[1,2]$, and use it to establish the precise equivalence between our results and theirs.

The conclusion - that chiral symmetry is broken by supersymmetric boundary 
conditions in ADS - is verified in Sect. four by showing that the order parameter $\langle\bar{\psi} \psi\rangle$ of chiral symmetry breaking does not vanish for either choice of supersymmetric boundary conditions. This confirms that Weyl spinors satisfying those boundary conditions do not exist on ADS.

\section{The Spinor Parallel Propagator $D_{A}^{A^{\prime}}\left(x, x^{\prime}\right)$}

In this paper the notation for two-component spinors and all signature and curvature conventions are those of Penrose and Rindler [5]. The only difference is that we use dotted and undotted spinor indices rather than primed and unprimed ones. In our paper a primed index indicates that it lives in the tangent space at $x^{\prime}$, unprimed indices live at $x$.

The use of two-component spinors restricts us to four dimensions and signature -2 . We also adopt the bitensors $n^{a}\left(x, x^{\prime}\right), n^{a^{\prime}}\left(x, x^{\prime}\right)$ and $g_{a}^{b^{\prime}}\left(x, x^{\prime}\right)$ as geometrically defined by Allen and Jacobson [4] for maximally symmetric spaces. However our conventions: $(+,-,-,-)$ and $2 \nabla_{[a} \nabla_{b]} V_{c}=-R_{a b c}{ }^{d} V_{d}$ are opposite those [4]. Nonetheless, all the formulae given in Table 1 of [4] remain correct, except that now $R^{2}>0$ for ADS and $\mu^{2}>0$ for timelike separated points, and the scalar curvature is $-12 / R^{2}$. [In the conventions of [4] $R^{2}>0$ for DS and $\mu^{2}>0$ for spacelike separations. $R$ denotes the radius of the space.] The formulae of that table are sufficient for our needs, and necessary to reproduce our calculations.

Our fundamental object is the bispinor $D_{A}{ }^{\prime}\left(x, x^{\prime}\right)$. It parallel transports a twospinor $\phi^{A}$ at the point $x$, along the shortest geodesic to the point $x^{\prime}$, yielding a new spinor $\chi^{A^{\prime}}$ at $x^{\prime}$,

$$
\chi^{A^{\prime}}=\phi^{A} D_{A}^{A^{\prime}}\left(x, x^{\prime}\right)
$$

Complex-conjugate spinors are similarly transported by the complex conjugate of $D_{A}{ }^{A^{\prime}}$, which is $\bar{D}_{\dot{A}}^{\hat{A}^{\prime}}$. The elementary properties of $D_{A}{ }^{A^{\prime}}$ are given in the appendix.

We need to find the covariant derivative of $D_{A}{ }^{A}$, which must be formed from the tangent to the geodesic $n_{A \dot{A}}=n_{a}$ and from $D_{A}{ }^{A^{\prime}}$ itself (the proof parallels Appendix B of $[4])$ :

$$
\nabla_{A \dot{A}} D_{B}{ }^{B^{\prime}}=\alpha(\mu) n_{A \dot{A}} D_{B}{ }^{B^{\prime}}+\beta(\mu) n_{B_{\dot{A}}} D_{A}{ }^{B^{\prime}} .
$$

Here $\mu\left(x, x^{\prime}\right)$ is the geodesic separation of $x$ and $x^{\prime}, n_{A \dot{A}}=\nabla_{A A} \mu, n_{A^{\prime} A^{\prime}}=\nabla_{A^{\prime} A^{\prime}} \mu$, and the functions $\alpha(\mu)$ and $\beta(\mu)$ remain to be determined.

Since $D_{B}{ }^{B^{\prime}}$ by definition satisfies

$$
n^{a} \nabla_{a} D_{B}^{B^{\prime}}=0
$$

and $n_{A \dot{A}} n^{B \dot{A}}=1 / 2 \delta_{A}{ }^{B}$, it follows that $\beta(\mu)=-2 \alpha(\mu)$. To determine $\beta(\mu)$ we use the Ricci identity (integrability condition) for spinors, which is [5],

$$
2 \nabla_{[a} \nabla_{b]} \phi^{C}=\left[\varepsilon_{\dot{A} \dot{B}} X_{A B D}{ }^{C}+\varepsilon_{A B} \Phi_{\dot{A} B D}^{C}\right] \phi^{D} \text {. }
$$

On a maximally symmetric space the Riemann tensor is

$$
R_{a b c d}=\frac{-1}{R^{2}}\left(g_{a c} g_{b d}-g_{a d} g_{b c}\right)
$$


and the curvature spinors [5] are

$$
\begin{gathered}
X_{A B C D}=-\frac{1}{2 R^{2}}\left(\varepsilon_{A C} \varepsilon_{B D}+\varepsilon_{A D} \varepsilon_{B C}\right), \\
\Phi_{A B C \dot{D}}=0 .
\end{gathered}
$$

Therefore $D_{A}{ }^{A^{\prime}}$ must satisfy

$$
4 \nabla_{[a} \nabla_{b]} D^{C C^{\prime}}=-\frac{1}{R^{2}}\left(\varepsilon_{A E} \varepsilon_{B}^{c}+\varepsilon_{A}{ }^{c} \varepsilon_{B E}\right) \varepsilon_{A \dot{B}} D^{E C^{\prime}}
$$

Inserting (2.2) for $\nabla_{a} D_{B}{ }^{B^{\prime}}$ and contracting with $\varepsilon^{A} c^{\varepsilon^{A \dot{B}}}$ and $\varepsilon^{B}{ }^{B} n^{A \dot{A}}$, we find that $\beta(\mu)$ must satisfy respectively

$$
\begin{aligned}
\beta^{\prime}+3 A(\mu) \beta+\beta^{2}-2 / R^{2} & =0, \\
\beta^{\prime}+A(\mu) \beta-1 / R^{2} & =0,
\end{aligned}
$$

where ' $=d / d \mu$ and $A(\mu)=R^{-1} \cot (\mu / R)$. (Note: $A$ is given in Table 1 of [4]; it is defined by $\nabla_{a} n_{b}=A\left[g_{a b}-n_{a} n_{b}\right]$ ).

The unique solution to $(2.8)$ which vanishes as $(\mu / R) \rightarrow 0$ is $\beta=-(A+C)$, where $C=-R^{-1} \csc (\mu / R)$. Therefore we find

$$
\nabla_{A \dot{A}}{D_{B}}^{B^{*}}=(A+C)\left[\frac{1}{2} n_{A \dot{A}} D_{B}^{B^{\prime}}-n_{B \dot{A}} D_{A}^{B^{\prime}}\right] .
$$

This result implies (1.22) in [4], because the parallel propagator for vector indices is $g_{a}^{b^{\prime}}=D_{A}^{B^{\prime}} \bar{D}_{\dot{A}}^{B^{\prime}}$. Equipped with (2.9), we can find the spinor 2-point functions.

\section{Massive Two-point Functions}

We denote a four-component Dirac spinor by

$$
\psi_{\alpha}=\left[\begin{array}{c}
\phi_{A} \\
\bar{\chi}_{\dot{A}}
\end{array}\right],
$$

where $\phi_{A}$ and $\bar{\chi}_{A}$ are a pair of two-component spinors. The Dirac equations of motion are (ref. [5] Eq. 4.4.66)

$$
\begin{aligned}
& \nabla_{A \dot{A}} \phi^{A}=\frac{-m}{\sqrt{2}} \bar{\chi}_{\dot{A}}, \\
& \nabla_{A}^{\dot{A}} \bar{\chi}_{\dot{A}}=\frac{m}{\sqrt{2}} \phi_{A},
\end{aligned}
$$

where $m$ is the mass.

There are two basic two-point functions, which are

$$
\begin{aligned}
& P^{A \dot{B}^{\prime}}=\left\langle\phi^{A}(x) \bar{\phi}^{\dot{B}^{\prime}}\left(x^{\prime}\right)\right\rangle=f(\mu) D_{A^{A}} \cdot n^{A^{\prime} \dot{B}^{\prime}}, \\
& {Q_{\dot{A}} \dot{B}^{\prime}}^{\prime}=\left\langle\bar{\chi}_{\dot{A}}(x) \phi^{\dot{B}^{\prime}}\left(x^{\prime}\right)\right\rangle=g(\mu) \bar{D}_{\dot{A}}^{\dot{B}^{\prime}} .
\end{aligned}
$$

We assume temporarily that $x$ and $x^{\prime}$ are spacelike separated so that the field operators in (3.3) anti-commute. The right-hand side of (3.3) is the most general 
maximally symmetric bispinor with the correct index structure. [Any other expressions with the correct index structure can be reduced to it via the equations in the appendix.] The functions $f$ and $g$ depend only upon the geodesic distance $\mu$. We will shortly see that other two-point functions like $\left\langle\bar{\chi}_{\dot{A}} \chi_{B^{\prime}}\right\rangle$ are entirely determined by $f$ and $g$.

The equations of motion (3.2) now imply that

$$
\begin{aligned}
& \nabla_{A A} P^{A^{\dot{B}^{\prime}}}=\frac{-m}{\sqrt{2}} Q_{\dot{A}}^{\dot{B}^{\prime}}, \\
& \nabla_{A}{ }^{\dot{A}} Q_{\dot{A}}^{\dot{B}^{\prime \prime}}=\frac{m}{\sqrt{2}} P_{A}^{\dot{B}^{\prime}} .
\end{aligned}
$$

Thus inserting (3.3a(b)) into (3.4a(b)), one obtains two coupled equations for the coefficient functions $f(\mu)$ and $g(\mu)$,

$$
\begin{array}{r}
f^{\prime}+\frac{3}{2}(A-C) f+\sqrt{2} m g=0, \\
g^{\prime}+\frac{3}{2}(A+C) g-\frac{m}{\sqrt{2}} f=0 .
\end{array}
$$

Combining these two equations, one obtains a single second-order equation for $f$,

$$
f^{\prime \prime}+3 A f^{\prime}+\left[m^{2}-\frac{9}{4} R^{-2}+\frac{3}{2} C(A-C)\right] f \equiv 0,
$$

where we have used $A^{\prime}=-C^{2}, C^{\prime}=-A C$ and $C^{2}-A^{2}=R^{-2}$.

Before finding $f$, let us return to the other possible two-point functions. The expectation value of $\langle\psi \bar{\psi}\rangle$ may be written in terms of 2-component spinors as

$$
\left\langle\psi_{\alpha} \bar{\psi}^{\beta^{\prime}}\right\rangle=\left[\frac{\left\langle\phi_{A} \chi^{B^{\prime}}\right\rangle}{\left\langle\bar{\chi}_{\dot{A}} \chi^{B^{\prime}}\right\rangle} \mid \frac{\left\langle\phi_{A} \phi^{\dot{B}^{\prime}}\right\rangle}{\left\langle\bar{\chi}_{\dot{A}} \bar{\phi}^{\dot{B}^{\prime}}\right\rangle}\right] .
$$

From the definitions (3.3) and equations of motions (3.2) and (3.5) it follows that the remaining two combinations are

$$
\begin{aligned}
\left\langle\phi^{A} \chi_{B^{\prime}}\right\rangle & =-2^{1 / 2} m^{-1} \nabla_{B^{\prime} \dot{B}^{\prime}} P^{A^{\dot{B}^{\prime}}}=g(\mu) D_{B^{\prime}}^{A}, \\
\left\langle\bar{\chi}_{\dot{A}} \chi_{B^{\prime}}\right\rangle & =-2^{1 / 2} m^{-1} \nabla_{B^{\prime} \dot{B}^{\prime}} Q_{\dot{A}}^{\dot{B}^{\prime}}=-f(\mu) D^{A}{ }_{B^{\prime}} n_{A \dot{A}} .
\end{aligned}
$$

Thus in terms of 2-component bispinors

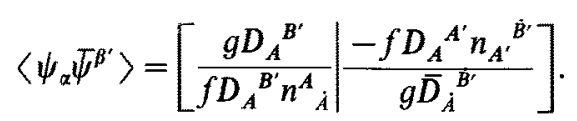

Now we solve for $f(\mu)$ and $g(\mu)$.

Changing variables to $Z=\cos ^{2}(\mu / 2 R)$, and letting

$$
w(Z)=\left[R^{2}(Z-1)\right]^{-1 / 2} f(Z),
$$

one obtains a hypergeometric equations for $w$,

$$
Z(1-Z) \ddot{w}+[c-(a+b+1) Z] \dot{w}-a b w=0,
$$

where $\cdot=d / d Z$. (Note: the factor $R^{2}$ is included in the definition of $w(3.10)$ to ensure that the standard branch cut of the square root lies along timelike separations 
$\mu^{2}>0$ ). The parameters $a, b$ and $c$ are

$$
a=2+\sqrt{m^{2} R^{2}}, \quad b=2-\sqrt{m^{2} R^{2}}, \quad c=2 .
$$

(Note: for $R$ real it is important that $a \geqq b$.) In the same way one may show that $Z^{-1 / 2} g$ satisfies a hypergeometric equation with parameters $a, b$, and $c+1$. We now need to specify boundary conditions to uniquely specify a solution to this equation.

The correct solution to (3.11) in de Sitter space $R^{2}<0$ is obtained [4] by demanding that it is only singular when $\mu=0$ and not when $\mu=\pi R$.

This yields

$$
\begin{aligned}
& f_{\mathrm{DS}}=N_{\mathrm{DS}}(1-Z)^{1 / 2} F(a, b ; c ; Z), \\
& g_{\mathrm{DS}}=-i N_{\mathrm{DS}} 2^{-3 / 2} m|R| Z^{1 / 2} F(a, b ; c+1 ; Z) .
\end{aligned}
$$

The short distance behavior $\mu \rightarrow 0$ can now be used to fix the constant $N_{\mathrm{DS}}$. In flat space $f \cong-i 2^{-1 / 2} \pi^{-2}\left(-\mu^{2}\right)^{-3 / 2}$ for small $\mu$, thus

$$
N_{\mathrm{DS}}=\frac{-i \Gamma(a) \Gamma(b)}{8 \sqrt{2} \pi^{2}|R|^{3}}=\frac{-i|R m|\left(1-m^{2} R^{2}\right)}{8 \sqrt{2} \pi|R|^{3} \sinh \pi|R m|} .
$$

The Feynman function is obtained by evaluating $f_{\mathrm{Ds}}(Z)$ and $g_{\mathrm{Ds}}(Z)$ above the branch cut from $Z=1$ to $\infty$, i.e. taking $f_{\mathrm{Ds}}(Z+i 0)$ and $g_{\mathrm{DS}}(Z+i 0)$. This concludes the de Sitter case.

In anti de Sitter space, it is necessary to impose boundary conditions at timelike spatial infinity, to (1) make the Cauchy problem well defined, and (2) conserve quantities (like the inner product between modes) formally conserved (via integration by parts) [4]. This leads to "reflecting" boundary conditions at spatial infinity, which for example reflect any flux of energy-momentum from the boundary at spatial infinity, and thus conserve the Hamiltonian. In supersymmetry these same boundary conditions also arise $[1,2]$.

There are two possible reflecting boundary conditions for a field of mass $m$. When $m$ is nonzero, these correspond to the two representations $D(3 / 2+|m R|, 1 / 2)$ and $D(3 / 2-|m R|, 1 / 2)$ of $\mathrm{S} 0(3,2)$. In the limit $m \rightarrow 0^{+}$, the two representations both become $D(3 / 2,1 / 2)$, but there are still two different two-point functions, because there are two physically inequivalent sets of modes that can from a representation of $D(3 / 2,1 / 2)$. These modes differ only by a parity assignment. Following [2] (p. 270) one set of modes is labeled $D(3 / 2,1 / 2)^{+}$and the other set of modes is labeled $D(3 / 2,1 / 2)^{-}$. The "minus" nodes are obtained from the "plus" modes by a chiral transformation whose effect is to change the sign of $\phi_{A}$, while leaving $\chi_{A}$ unchanged. The correlation functions of the modes in these two sets can be obtained as the limit $m \rightarrow 0^{+}$of the two distinct massive representations given above.

In the representation $D(3 / 2+|m R|, 1 / 2)$, which we label by " + ", the modes, and the two-point function, fall off as fast as possible at spatial infinity $Z \rightarrow \infty$. (This turns out to imply that $f$ and $g$ are singular at both $Z=0$ and $Z=1$.) Since $a>b$, we therefore obtain

$$
\begin{gathered}
f_{\mathrm{ADS}}^{+}=N_{\mathrm{ADS}}^{+}(Z-1)^{1 / 2} Z^{-a} F\left(a, a-c+1 ; a-b+1 ; Z^{-1}\right), \\
g_{\mathrm{ADS}}^{+}=i \frac{m}{|m|} N_{\mathrm{ADS}}^{+} 2^{-1 / 2} Z^{1 / 2-a} F\left(a, a-c ; a-b+1 ; Z^{-1}\right),
\end{gathered}
$$


and the constant is

$$
N_{\mathrm{ADS}}^{+}=\frac{-i \Gamma(a) \Gamma(a-1)}{8 \sqrt{2} \pi^{2}|R|^{3} \Gamma(a-b+1)} .
$$

The reason that the factor $m /|m|$ has appeared in the formula for $g^{+}$, is that changing the sign of $m$ (see (3.2)) is equivalent to changing the sign of spinor $\phi_{A}$, and this has the effect of changing the sign of (3.3b), and hence of $g(\mu)$. In the limit $m \rightarrow 0^{+}, f^{+}$and $g^{+}$give the two-point function of the modes in $D(3 / 2,1 / 2)^{+}$. In general, the Feynman function is obtained by evaluating $f_{\text {ADS }}^{+}$and $g_{\text {ADs }}^{+}$just above the branch cut from $Z=0$ to 1 , i.e. taking $f_{\mathrm{ADS}}(Z+i 0)$. We will return to the representation $D(3 / 2-|m R|, 1 / 2)$ later.

\section{Massless Two-point Functions and Chiral Symmetry Breaking}

On de Sitter space the massless four-component spinor correlation functions are given by (3.13)

$$
\begin{aligned}
& f_{\mathrm{DS}}=\frac{-i}{8 \sqrt{2} \pi^{2}|R|^{3}}(1-Z)^{-3 / 2} \\
& g_{\mathrm{DS}}=0
\end{aligned}
$$

The function $f_{\mathrm{DS}}$, and thus $\left\langle\phi^{A} \bar{\phi}^{\dot{B^{\prime}}}\right\rangle$, is singular in the coincidence limit $(\mu \rightarrow 0$ or $Z \rightarrow 1$ ) as in flat space. Because the function $g_{\mathrm{Ds}}$, and thus $\left\langle\bar{\chi}_{\dot{A}} \phi^{\dot{B}^{\prime}}\right\rangle$, is identically zero, there is no correlation between the right-and left-handed components $\phi$ and $\chi$. Thus the massless four-component Dirac spinor on de Sitter space is equivalent to two decoupled massless two-component Weyl spinors.

This result is not true on anti de Sitter space because the boundary conditions are different there. From (3.15), when $m \rightarrow 0^{+}$, the two-point function for the modes in $D(3 / 2,1 / 2)^{+}$becomes

$$
\begin{aligned}
f^{+}{ }_{\mathrm{ADS}} & =\frac{-i}{8 \sqrt{2} \pi^{2}|R|^{3}}(Z-1)^{-3 / 2} \\
g^{+}{ }_{\mathrm{ADS}} & =\frac{1}{16 \pi^{2}|R|^{3}} Z^{-3 / 2} .
\end{aligned}
$$

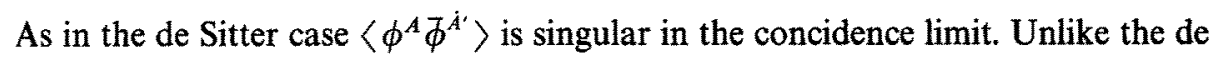
Sitter case, the function $g^{+}$is nonzero, and is singular in the limit $Z \rightarrow 0$, when $x$ and $x^{\prime}$ are antipodal points. Hence the right and left components of the four-component massless spinor are not uncorrelated on anti de Sitter space. Thus we conclude that there exist no Weyl spinors that satisfy appropriates ADS boundary conditions because the existence of such spinors would force $g^{+}(Z)$ to vanish.

Breitenlohner and Freedman [1] have observed that supersymmetric boundary conditions on ADS break the chiral U(1) invariance of the linearized spin-0/spin-1/2 sector of gauged $N=4$ supergravity. Weyl spinors are therefore absent because supersymmetric boundary conditions break chiral symmetry. In a similar vein, 
Fronsdal [8] has pointed out that right- and left-handed neutrinos in ADS are distinguished by a superselection rule.

We have already discussed the representation $D(3 / 2+|m R|, 1 / 2)$ for a field of mass $m$. The other possible representation for a field of mass $m$ is $D(3 / 2-|m R|, 1 / 2)$. In this representaion we call the two-point function $f^{-}, g^{-}$. It is

$$
\begin{aligned}
& f_{\mathrm{ADS}}^{-}=N^{-}{ }_{\mathrm{ADS}}(Z-1)^{1 / 2} Z^{-b} F\left(b, b-c+1, b-a+1, Z^{-1}\right), \\
& g^{-}{ }_{\mathrm{ADS}}=-i 2^{-1 / 2} \frac{m}{|m|} N^{-}{ }_{\mathrm{ADS}} Z^{1 / 2-b} F\left(b, b-c, b-a+1, Z^{-1}\right),
\end{aligned}
$$

with

$$
N^{-}{ }_{\mathrm{ADS}}=\frac{-i \Gamma(b) \Gamma(b-1)}{8 \sqrt{2} \pi^{2}|R|^{3} \Gamma(b-a+1)} .
$$

Thus in the massiess limit $m=0^{+}$, this yields

$$
\begin{aligned}
& f_{\mathrm{ADS}}^{-}=\frac{-i}{8 \sqrt{2} \pi^{2}|R|^{3}}(Z-1)^{-3 / 2}, \\
& g_{\mathrm{ADS}}^{-}=\frac{-1}{16 \pi^{2}|R|^{3}} Z^{-3 / 2},
\end{aligned}
$$

which is identical to (4.2) except for the sign of $g$. Thus chiral symmetry is broken for both the $D(3 / 2,1 / 2)^{+}$modes and the $D(3 / 2,1 / 2)^{-}$modes.

There is a simple way to understand the change of sign between $g^{+}$and $g^{-}$when $m=0$. As discussed earlier, the modes in $D(3 / 2,1 / 2)^{+}$are obtained from those in $D(3 / 2,1 / 2)^{-}$by a chiral transformation (ref. [1] pg. 270), whose effect is to change the sign of $\phi_{A}$ while leaving $\chi_{A}$ unaffected. Hence the expectation value $\left\langle\phi_{A} \bar{\phi}^{\dot{B}^{\prime}}\right\rangle$ (and thus $f$ ) is unchanged, but the expectation value $\left\langle\phi_{A} \chi^{B^{\prime}}\right\rangle$ (and thus $g$ ) changes sign.

As a final check of our conclusions, we calculate the order parameter $\langle\bar{\psi} \psi\rangle=$ trace $\langle\psi \bar{\psi}\rangle$. This quantity is not invariant under chiral transformations $\psi \rightarrow e^{i \alpha} \gamma_{5} \psi$, and thus serves as an index of chiral symmetry breaking. By the trace of $\langle\psi \bar{\psi}\rangle$ we mean

$$
\begin{aligned}
& \langle\bar{\psi} \psi\rangle=\lim _{x^{\prime} \rightarrow x} \operatorname{trace}\left\langle\psi(x) \bar{\psi}\left(x^{\prime}\right)\right\rangle \\
& =\lim _{x^{\prime} \rightarrow x}\left[\left\langle\phi_{A} \chi^{B^{\prime}}\right\rangle D_{B^{\prime}}{ }^{A}+\left\langle\bar{\chi}_{A} \bar{\phi}^{\dot{B}^{\prime}}\right\rangle \bar{D}_{\dot{B}}{ }^{\dot{ }}\right]=\lim _{x^{\prime} \rightarrow x} 4 g(\mu),
\end{aligned}
$$

and in the coincidence limit $Z \rightarrow 1$ one obtains from (4.2b) and (4.5b) respectively,

$$
\langle\bar{\psi} \psi\rangle=4 g_{\text {ADS }}^{ \pm}(Z=1)= \pm\left(4 \pi^{2}\right)^{-1}|R|^{-3}
$$

for the modes in $D(3 / 2,1 / 2)^{ \pm}$. We stress that this method of calculation requires no regularization of any kind.

We can also calculate $\langle\bar{\psi} \psi\rangle$ using dimensional regularization, by taking the coincidence limit, and tracing for $n \neq 4$ and then analytically continuing to $n=4$. Following Appendix B of [2] we obtain, for the modes in $D(3 / 2+m R, 1 / 2)^{ \pm}$, 


$$
\langle\bar{\psi}\rangle= \pm(2 \pi)^{-n / 2}|R|^{-3}\left(m R+\frac{n-2}{2}\right) \frac{\Gamma(1-n / 2) \Gamma(m R+n / 2-1)}{\Gamma(m R+1-n / 2)} .
$$

In the massless limit $m=0$, where we might except chiral symmetry to be restored, we obtain the same result (4.7) as above. This confirms our conclusion.

\section{The Bispinor $S(x) \bar{S}\left(x^{\prime}\right)$, and the Representations $D(3 / 2 \pm|m R|, 1 / 2)$}

In this section we establish the precise connection between the spinor two-point functions of [2] and the two-point functions in this paper. To do this, we need to express the (4-component) bispinor $S(x) \bar{S}\left(x^{\prime}\right)$ defined in [1] in terms of geometrical objects. Reference [2] notes that if $G\left(x, x^{\prime}\right)$ satisfies the scalar wave equation for mass $M_{\text {scalar }}^{2}=m^{2}-m / R-2 / R^{2}$, then

$$
\left\{\left[i \partial_{x}+m+1 / R\right] G\left(x, x^{\prime}\right)\right\} S(x) \bar{S}\left(x^{\prime}\right)
$$

satisfies the Dirac equation for mass $m$. We will use this fact to find $S(x) \bar{S}\left(x^{\prime}\right)$.

First note that if $m=0$ or $m=1 / R$, then the scalar mass takes the conformally invariant value $M_{\text {scalar }}^{2}=-2 / R^{2}$. Secondly, if $G\left(x, x^{\prime}\right)$ has only one singular point as $Z \rightarrow 1$, then (5.1) also has only one singular point (the converse is also true). Thus choosing $G\left(x, x^{\prime}\right)$ to be the conformally invariant scalar two-point function with only one singular point $((2.9)[4])$

$$
G=\frac{1}{16 \pi^{2} R^{2}}(Z-1)^{-1}
$$

we know from (5.1) that

$$
\begin{aligned}
\langle\psi \bar{\psi}\rangle_{m=1 / R} & =\{(i \not \partial+2 / R) G\} S x) \bar{S}\left(x^{\prime}\right), \\
\langle\psi \bar{\psi}\rangle_{m=0} & =\{(i \not \partial+1 / R) G\} S(x) \bar{S}\left(x^{\prime}\right),
\end{aligned}
$$

where $\langle\psi \bar{\psi}\rangle$ are correlation functions with only one singular point on ADS. Thus subtracting (5.3b) from (5.3a) we find

$$
S(x) \bar{S}\left(x^{\prime}\right)=R G^{-1}\left[\langle\psi \bar{\psi}\rangle_{m=1 / R}-\langle\psi \bar{\psi}\rangle_{m=0}\right] .
$$

Defining $\Delta f=f_{m=1 / R}-f_{m=0}$ and $\Delta g$ similarly, and using (3.13), one obtains

$$
\begin{aligned}
& \Delta f=\frac{i}{8 \sqrt{2} \pi^{2} R^{3}}(Z-1)^{-1 / 2}, \\
& \Delta g=\frac{1}{16 \pi^{2} R^{3}} Z^{1 / 2}(Z-1)^{-1} .
\end{aligned}
$$

Thus from (3.9) and (5.4) we find

$$
\left[S(x) \bar{S}\left(x^{\prime}\right)\right]_{\alpha}^{\beta^{\prime}}=\left[\frac{Z^{1 / 2} D_{A}^{B^{\prime}}}{i \sqrt{2}(Z-1)^{1 / 2} D_{C}^{B^{\prime}} n^{C}{ }_{\dot{A}}} \mid \frac{-i \sqrt{2}(Z-1)^{1 / 2} D_{A}^{C^{\prime}} n_{C^{B^{\prime}}}^{\dot{\beta}^{\prime}}}{Z^{1 / 2} \bar{D}_{\dot{A}}^{B^{\prime}}}\right] .
$$

In the limit as $x \rightarrow x^{\prime}$ (or $Z \rightarrow 1$ ) this reduces to the identity operator, as expected. Having obtained an expression for $S(x) \bar{S}\left(x^{\prime}\right)$ we can now compare our two-point 
functions to those of [2]. Take for $\gamma$-matrices [6],

$$
\left(\gamma_{p}\right)_{\alpha}{ }^{\beta}=-i \sqrt{2}\left[\frac{0}{\varepsilon_{\dot{P} \dot{A}} \varepsilon_{P}{ }^{B}} \mid \frac{\varepsilon_{P A} \varepsilon_{\dot{P}}{ }^{\dot{B}}}{0}\right] .
$$

In this basis, the Dirac equation $[i \not-m] \psi=0$ with $\psi$ given by (3.1) implies the equations of motion (3.2) for $\phi_{A}$ and $\bar{\chi}_{\dot{A}}$. Now if $G(\mu)$ is any function of geodesic distance, then

$$
i \phi_{x} G(\mu)=i \gamma_{p} n^{P} G^{\prime}(\mu)=\sqrt{2}\left[\frac{0}{n_{A}^{B} G^{\prime}(\mu)} \mid \frac{n_{A}{ }^{\dot{B}} G^{\prime}(\mu)}{0}\right] .
$$

Thus combining (5.1) with (5.6) and (5.8), one obtains for $\langle\psi \bar{\psi}\rangle$ the expression given in (3.9), with

$$
\begin{aligned}
& f=\frac{i \sqrt{2}}{R}(Z-1)^{1 / 2}\left[Z \frac{d}{d Z}+(1-m R)\right] G(Z), \\
& g=\frac{Z^{1 / 2}}{R}\left[(Z-1) \frac{d}{d Z}+(1+m R)\right] G(Z),
\end{aligned}
$$

where we have used $d Z / d \mu=i R^{-1}\left(Z^{2}-Z\right)^{1 / 2}$.

Now we can compare our two-point functions with those of [2]. In that reference, we first take the scalar field $A$ in $([2], 7.10)$ to have a mass parameter $\lambda=$ $1+|m R|$. Then the two-point function for $A$ is

$$
G(Z)=(4 \pi R)^{-2} \frac{\Gamma(\lambda) \Gamma(\lambda-1)}{\Gamma(2 \lambda-2)}(Z-1)^{-\lambda} F\left(\lambda, \lambda-1 ; 2 \lambda-2 ;(1-Z)^{-1}\right) .
$$

[Note: in the notation of [2] $u=2(1-Z)$.] From (5.9) and (5.10) we can easily see [7] that this $G(Z)$ of Burges et al. [2] gives our $f_{\mathrm{ADS}}^{+}$and $g^{+}{ }_{\mathrm{ADS}}$ of (3.15). Thus $f^{+}, g^{+}$and the representation $D(3 / 2+|m R|, 1 / 2)$ corresponds to choosing the regular representation of $A$ for $|m R|>1 / 2$ and the irregular representation of $A$ for $|m R|<1 / 2$. In the massless limit $\psi$ belongs to $D(3 / 2,1 / 2)^{+}$.

Similarly, if we take the scalar field $A$ to have a mass parameter $\lambda=2-|m R|$, this gives the two-point function $f^{-}, g^{-}(4.3)$ and $\psi$ lies in $D(3 / 2-|m R|, 1 / 2)$. Thus, $f^{-}, g^{-}$ corresponds to choosing the (nonunitary) irregular representation of $A$ for $|m R|>1 / 2$ and the regular representation of $A$ for $|m R|<1 / 2$. In the massless limit $\psi$ belongs to $D(3 / 2,1 / 2)^{-}$.

We have also confirmed formula (7.10) of reference [2] by carrying out a bruteforce sum of the spinor mode functions. Since that lengthy calculation simply confirms the results given there, we have not included it here; details are available from the authors. We have also recently learned that further remarks by Freedman on the boundary conditions in anti de Sitter space may be found in [9].

\section{Appendix. Properties of the Parallel Propagator $D_{A}{ }^{A}$}

$$
\begin{aligned}
D_{A}{ }^{\prime}\left(x, x^{\prime}\right) & =-D^{A^{\prime}}{ }_{A}\left(x^{\prime}, x\right) \text { or } D_{A}^{A^{\prime}}=-D^{A^{\prime}}{ }_{A}, \\
D_{A}{ }^{A^{\prime}} D_{A^{\prime}}{ }^{B} & =\varepsilon_{A}{ }^{B}, \\
D_{A A^{\prime}} D^{A A^{\prime}}=2 &
\end{aligned}
$$




$$
\begin{aligned}
& \left(\lim _{x \rightarrow x^{\prime}} D_{A}^{B^{\prime}}\right)=\varepsilon_{A}{ }^{B}, \\
& \left(\lim _{x \rightarrow x^{\prime}} D_{A B^{\prime}}\right)=\varepsilon_{A B}, \\
& g_{a}^{b^{\prime}}=D_{A}{ }^{B^{\prime}} \bar{D}_{\dot{A}}^{\dot{B}^{\prime}}, \\
& D_{A}{ }^{B^{\prime}} \bar{D}_{\dot{A}}^{\dot{B}^{\prime}} n^{A \ddot{A}}=-n^{B^{\prime} \dot{B}^{\prime}}, \\
& n_{A \dot{C}} D_{B^{\prime}}^{A}=-n_{B^{\prime} B^{\prime}} \bar{D}_{{ }_{C}^{\prime}}^{\dot{B}^{\prime}} \\
& \nabla_{A \dot{A}} D_{A^{\prime}}=(3 / 2)(A+C) n_{A \dot{A}} D_{A^{\prime}}^{A} \text {, } \\
& D^{A}{ }_{A^{\prime}} \nabla_{A A^{\prime}} n^{A^{\prime} \dot{A}^{\prime}}=-\frac{3}{2} C \bar{D}_{\dot{A}}^{\dot{A}^{\prime}} \text {, } \\
& \nabla_{A \dot{A}} D_{B}{ }^{B^{\prime}}=(A+C)\left[\frac{1}{2} n_{A \dot{A}} D_{B}^{B^{\prime}}-n_{B \dot{A}} D_{A}^{B^{\prime}}\right], \\
& \nabla_{A \dot{A}} n_{\dot{B}}^{A}=\frac{3}{2} A \varepsilon_{\dot{A} \dot{B}} \text {. }
\end{aligned}
$$

Acknowledgements. We are grateful for discussions with C. J. C. Burges, P. Candelas, D. Z. Freedman, G. W. Gibbons and T. Jacobson. This work was supported in part by NSF grants PHY82-05717, PHY81-07384, PHY83-51860, a University of Texas travel grant, the Norwegian Council for Science and Humanities (NAVF), and the General Electric Company.

\section{References}

1. Breitenlohner, P., Freedman, D. Z:: Stability in gauged extended supergravity. Ann. Phys. 144, 249$281(1982)$

2. Burges, C. J. C., Davis, S., Freedman, D. Z., Gibbons, G. W.: Supersymmetry in anti de Sitter space. Ann. Phys. 167, 285-316 (1986)

3. Gibbons, G. W., Hawking, S. W., Siklos, S. T. C. (eds.): The very early Universe. Cambridge: Cambridge University Press 1983

4. Allen, B., Jacobson, T.: Vector two-point functions in maximally symmetric spaces. Commun. Math. Phys. 103, 669-692 (1986)

5. Penrose, R., Rindler, W.: Spinors and space-time. Cambridge: Cambridge University Press 1984

6. These are the same $\gamma$-matrices given by [5] in the long footnote on p. 221 except for a factor of $-i$. That factor is necessary since [5] assumes $\left\{\gamma_{a}, \gamma_{b}\right\}=-2 g_{a b}$ and [2] assumes $\left\{\gamma_{a}, \gamma_{b}\right\}=2 g_{a b}$

7. The quickest way to establish that the solutions are the same is to show that they have the same asymptotic fall off as $Z \rightarrow \infty$, and the same singular behavior at $Z=1$. Since one may show that they both satisfy the same second-order ordinary differential (3.6), they are therefore equal

8. Fronsdal, C.: Elementary particles in curved space, IV. Massless particles. Phys. Rev. D12, 3819-3830 (1975)

9. Freedman, D. Z.: A fond farewell to anti de Sitter space. In: Proceedings of the Cambridge supersymmetry workshop 1985. Gibbons, Hawking, Townsend (eds.) Cambridge: Cambridge University Press 1986

Communicated by A. Jaffe

Received January 10, 1986; in revised form February 28, 1986 